\title{
The $c g$-average tree value for games on cycle-free fuzzy communication structures
}

\author{
J. R. Fernández ${ }^{2} \cdot$ I. Gallego ${ }^{3} \cdot$ A. Jiménez-Losada ${ }^{1}$ (I) $\cdot$ M. Ordóñez ${ }^{2}$ \\ Received: 6 July 2018 / Accepted: 14 May 2019 / Published online: 6 June 2019 \\ c Sociedad de Estadística e Investigación Operativa 2019
}

\begin{abstract}
The main goal in a cooperative game is to obtain a fair allocation of the profit due the cooperation of the involved agents. The most known of these allocations is the Shapley value. This allocation considers that the communication among the players is complete. The Myerson value is a modification of the Shapley value considering a communication structure which determines the feasible bilateral relationships among the agents. This allocation of the profit is not always a stable solution. Another payoff allocation for games with a communication structure from the definition of the Shapley value is the average tree value. This one is a stable solution for any game using a cycle-free communication structure. Later fuzzy communication structures were introduced. In a fuzzy communication structure, the membership of the agents and the relationships among them are leveled. The Myerson value was extended in several different ways depending on the behavior of the agents. In this paper, the average tree value is extended to games with fuzzy communication structures taking one particular version: the Choquet by graphs $(\mathrm{cg})$. We present an application to the management of an electrical network with an algorithmic implementation.
\end{abstract}

Keywords Game theory · Fuzzy graph · Shapley value · Fuzzy communication structure

Mathematics Subject Classification 91A12 - 05C72 - 91A43 · 91A06

$凶 \quad$ A. Jiménez-Losada

ajlosada@us.es

1 Escuela Técnica Superior de Ingenieros, Matemática Aplicada II, Universidad de Sevilla, Camino de los Descubrimientos, 41092 Sevilla, Spain

2 Escuela Politécnica Superior, Matemática Aplicada II, Universidad de Sevilla, Virgen de África 7, 41011 Sevilla, Spain

3 Facultad de Ciencias de la Educación, Didáctica de las Matemáticas, Universidad de Sevilla, 41013 Sevilla, Spain 


\section{Introduction}

The main mathematical goal of this paper is to extend the average tree value (Herings et al. 2008) for games restricted by fuzzy communication situations without cycles. Using the Choquet partition (Choquet 1953), we introduce the $c g$-average value for cycle-free fuzzy communication games, in short AF value, analyzing its axiomatization and algorithmic implementation. Game theory was used in Hobbs (1992) and Contreras (1997) to analyze electrical networks. The motivation of this article is to give a costsharing rule for the client nodes in an electric partially interconnected network, which is supplied with energy by a power plant. The peculiarity of the network is that nodes do not need the same amount of energy and transmission channels do not have the same transmission capacity. Indeed, they stop working when their maximum transmission capacity is reached. The $c g$-average tree value allows us to achieve this sharing of costs among the nodes.

A cooperative game with transferable utility over a finite set of players is a mapping that assigns a real number to each subset of the power set of players, named coalitions. A payoff vector is a vector in which each component represents the payment for each player generated due to his cooperation possibilities. The Shapley value (Shapley 1988 ) is the best known of these outcomes and the first to be defined. This payoff vector supposes that all the communications are feasible. Later, Myerson (1977) describes the communication situation by a graph where the vertices are the players and the links are the feasible bilateral communications among them. This graph is named the communication structure of the game. So, Myerson proposed a more realistic situation for the game and a payoff vector for each communication structure. A communication value assigns a payoff vector to each game with a specific communication structure. The Myerson value extends the Shapley value in the sense that both coincide when the graph is complete. The complexity of computing the Myerson value has been studied in Bilbao et al. (2002) and Fernández et al. (2002). Another value for games with communication structure is the average tree value (Herings et al. 2008, 2010). The average tree value considers that the sets of feasible coalitions follow from a permission structure (Gilles et al. 1992) on the set of players, in which players need permission to cooperate with other players. An oriented tree can be seen as a permission structure. This means that there is one player at the top of the permission structure and for every other player there is a unique directed path from the top player to this player.

Aubin (1981) studied fuzzy coalitions, introducing vagueness about the membership of the players. To calculate the worth of a fuzzy coalition in a game, it is necessary to consider a specific partition by levels of this fuzzy set. One of these partitions was defined in Tsurumi et al. (2001) using the Choquet integral. Recently, we introduced in Jiménez-Losada et al. (2010) fuzzy graphs to analyze communication among players. The idea of partition by levels was extended to fuzzy communication structures in Jiménez-Losada et al. (2013), proposing different extensions of the Myerson value for fuzzy situations. In one of them, the Choquet by graph or $c g$ option, players look for the biggest communication structure at the greatest level at every moment.

In Sect. 2, we introduce a background about cooperative games and communication structures which allows the reader to follow the paper. Games with fuzzy communication structure and the $c g$-partition, the technical elements to introduce our value are 
studied in Sect. 3. Section 4 is dedicated to defining the $c g$-average value. In Sect. 5, we obtain an axiomatization of the value and we study its stability. In Sect. 6, we describe the algorithm $A F$ value to compute the $c g$-average value and we study its computational complexity. The conclusions are in the Sect. 7. We think that this study can be a preliminary work to continue with these applications.

\section{Preliminaries}

\subsection{Graphs}

Given a finite set $N$, we denote by $L N=\{\{i, j\}: i, j \in N, i \neq j\}$ the set of unordered pairs of different elements in $N$. A graph is a pair $g=(N, L)$, where $N$ is a finite set of elements (named vertices or nodes) and $L \subseteq L N$ is a subset of pairs (named edges or links). We use $i j$ to represent the pair $\{i, j\}$. Let $g=(N, L)$ be a graph. A sequence of $k$ different vertices $\left(i_{1}, \ldots, i_{k}\right)$ is a path in $g$ if $i_{l} i_{l+1} \in L$ for $l=1, \ldots, k-1$. The graph is connected if for all $i, j \in N$ there is a path $\left(i_{1}, \ldots, i_{k}\right)$ in which $i_{1}=i$ and $i_{k}=j$. We say that $g$ is cycle-free or forest if for every two different connected vertices $i, j \in N$, there is at most a unique path connecting $i$ and $j$. If the graph is connected and cycle-free, then it is named tree. A tree with $n$ vertices has $n-1$ edges. If an edge is removed from a tree, then the resulting graph is not connected. If a single edge is added to a tree, then the resulting graph contains a single cycle. If $S \subset N$ then $g_{S}=(S, L(S))$ with $L(S)=\{i j \in L: i, j \in S\}$ is a new graph named the subgraph induced by $S$. Set $S$ is connected in $g$ if the graph $g_{S}$ is connected. A connected component of $g$ is a maximal connected subgraph contained in it. A bridge is an edge whose elimination increases the number of components. For any set $S$, we name components of $S$ in $g$ the subsets in the family $S / g=\left\{R \subseteq S: g_{R}\right.$ connected component of $\left.g_{S}\right\}$. Family $S / g$ is a partition of $S$.

Let $g=(N, L)$ be a tree and consider any vertex $p$ of $g$, the selection of the vertex $p$, called root, allows the introduction of the following relation of order between the nodes of $g: i \leq j$ if, and only if, there exists a path from $p$ to $j$ which contains vertex $i$. Vertex $p$ is the first element in this situation. Tree $g$ endowed with the order of vertex $p$ is often referred as a tree with root $p$, and is denoted $g^{p}$. Consider a node $i$ in a rooted tree $g^{p}$. Any vertex $j$ on the unique path from $p$ to $i$ is called an ancestor of $i$. If $j$ is an ancestor of $i$, then $i$ is a descendant of $j$. Every vertex is both an ancestor and a descendant of itself. If $j$ is an ancestor of $i$ and $i \neq j$, then $j$ is a proper ancestor of $i$ and $i$ is a proper descendant of $j$. The subtree rooted at $i$ is the tree induced by descendant of $i$, rooted at $i$. Fixed a rooted tree $g^{p}$, the rooted subtree at $i$ is denoted by $g_{i}^{p}$. The set of descendants of vertex $i$ is $C^{g^{p}}(i)$, notice that $C^{g^{p}}(p)=N$. If the last edge in the unique path from the root $p$ to a node $j$ is $i j$ then $i$ is the parent of $j$, and $j$ is a child of $i$. The set of children of the vertex $j$ in the rooted subtree $g_{j}^{p}$ is denoted by $N^{g^{p}}(j)$. The root is the only vertex in $g^{p}$ with no parent. A node with no children is a leaf. The number of children of a node $i$ in a rooted tree is called the degree of $i$. The length of the path from the root $p$ to a node $i$ is the depth of $i$ in the tree. The height of a node in a tree is the number of edges on the longest simple downward path from the node to a 
leaf, and the height of a tree is the height of its root. The height of a tree is also equal to the largest depth of any node in the tree, for more details, see Cormen et al. (2001).

\subsection{Cooperative games}

A cooperative game with transferable utility, game for short, is a pair $(N, v)$, where $N$ is a finite set of $n$ players and $v: 2^{N} \rightarrow \mathbb{R}$ with $v(\emptyset)=0$ is named the characteristic function. A subset $S \in 2^{N}, S \neq \emptyset$, is called a coalition. For any coalition $S, v(S)$ displays the worth of coalition $S$. In all the paper, we consider a set of players $N=$ $\{1, \ldots, n\}$ fixed and then we identify a game with a characteristic function over $N$. We denote as $G^{N}$ the family of games over $N$.

A payoff vector of a game $v \in G^{N}$ is a vector $x \in \mathbb{R}^{N}$ giving a payoff $x_{i}$ to every player $i \in N$. The core (Gillies 1953) of the game $v$ is the set of stable and efficient payoff vectors, namely

$$
\operatorname{Core}(v)=B i g\left\{x \in \mathbb{R}^{N}: x(N)=v(N), x(S) \geq v(S), \forall S\right\}
$$

The core is defined supposing that the worth $v(S)$ is understood as a profit. A value for games over $N$ is a mapping $f: G^{N} \rightarrow \mathbb{R}^{N}$ that assigns to every game a payoff vector. A value $f$ is efficient if

$$
\sum_{i \in N} f_{i}(v)=v(N)
$$

for every $v \in G^{N}$. The best-known efficient value for games is the Shapley value (Shapley 1988) defined for any $v \in G^{N}$ and $i \in N$ as

$$
\phi_{i}(v)=\sum_{T \subseteq N \backslash\{i\}} \frac{t !(n-t-1) !}{n !}[v(T \cup i)-v(T)],
$$

where $t=|T|$. The Shapley value is not always in the core. The reader can use (Driessen 1988) for a deep knowledge about cooperative games.

\subsection{Games and communication structures}

Myerson (1977) introduced communication structures for games in 1977 to analyze situations in a game depending on the feasible coalitions of the players. Given our fixed set $N$ of players, a communication structure over $N$ is a graph $g=(N, L)$, where the nodes are the players and $L \subseteq L N$ represents the feasible bilateral communications among them. So, we will use graph or communication structure alike from here on. The family of communication structures over $N$ is denoted as $C S^{N}$. The communication structure $g$ is connected, non connected, cycle-free or tree if such is the graph.

A communication game over $N$ is a pair $(v, g)$ with $v \in G^{N}$ and $g \in C S^{N}$. We denote the collection of all the communication games as $C G^{N}$ and the class of all 
cycle-free communication games by $C G_{\mathrm{cf}}^{N}$. A communication value $f$ on a subclass of $C G^{N}$ assigns a unique payoff vector $f(v, g) \in \mathbb{R}^{N}$ to every $(v, g)$ in the subclass. Efficiency takes into account the components of the great coalition.

Efficient by components A communication value $f$ is efficient by components if for any $(v, g) \in C G^{N}$ it holds that

$$
\sum_{i \in K} f_{i}(v, g)=v(K)
$$

for every $K \in N / g$.

Let $(v, g) \in C G^{N}$. Myerson (1977) proposed the restricted game $v^{g} \in G^{N}$, defined by

$$
v^{g}(S)=\sum_{R \in S / g} v(R)
$$

for every coalition $S$, to introduce the information of the communication structure into the characteristic function. An example of communication value is the Myerson value (Myerson 1977) defined as $\mu(v, g)=\phi\left(v^{g}\right)$, for every $(v, g) \in C G^{N}$. But this value is not always in the core of the restricted game as it would be desirable.

Herings et al. (2008) introduced the average tree value, the AT value for short, as a communication value only on the cycle-free communication structures over $N$. Let $(v, g) \in C G_{\mathrm{cf}}^{N}$, suppose first that $g=(N, L)$ is a tree. So, we get the following payoff vector associated with player $j$ [the hierarchical outcomes introduced in Demange (2004)],

$$
t_{j}^{i}(v, g)=v\left(C^{g^{i}}(j)\right)-\sum_{u \in N^{g^{i}}(j)} v\left(C^{g^{i}}(u)\right), \quad \forall j \in N
$$

The AT value is defined by

$$
A T_{j}(v, g)=\frac{1}{|N|} \sum_{i \in N} t_{j}^{i}(v, g)
$$

If $g$ is cycle free but disconnected then we repeat the process inside each connected component. This value is a component efficient communication value characterized by component fairness.

Component fairness If $f$ is communication value and $(v, g) \in C G_{\mathrm{cf}}^{N}$ with $g=(N, L)$, then for all $K \in N / g$ and $i j \in L(K)$ it holds

$$
\frac{1}{\left|K_{i}^{i j}\right|} \sum_{h \in K_{i}^{i j}}\left[f_{h}(v, g)-f_{h}(v, g \backslash i j)\right]=\frac{1}{\left|K_{j}^{i j}\right|} \sum_{h \in K_{j}^{i j}}\left[f_{h}(v, g)-f_{h}(v, g \backslash i j)\right],
$$

Observe that as $g$ is cycle free, then we obtain a partition of $K$ in $g \backslash i j=(N, L \backslash i j)$ in two sets, $K_{i}^{i j}$ containing $i$ and $K_{j}^{i j}$ containing $j$. Hence, component fairness says that if a link is missing then the average of the payoff losses is the same for both new connected components. 
Fig. 1 Fuzzy graph $\gamma$

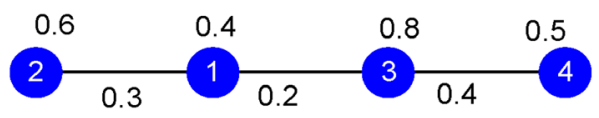

\section{Fuzzy communication structures}

Let $P$ be a finite set. A fuzzy set in $P$ is a function $\tau: P \rightarrow[0,1]$. The family of fuzzy sets in $P$ is denoted as $[0,1]^{P}$. Each subset $Q \subseteq P$ is associated with the fuzzy set $e^{Q} \in[0,1]^{P}$ with $e^{Q}(i)=1$ if $i \in Q$ and $e^{Q}(i)=0$ otherwise. Specifically, we denote $e^{\emptyset}=0$. The support of $\tau$ is $\operatorname{supp}(\tau)=\{i \in P: \tau(i) \neq 0\}$ and the image of $\tau$ is the set $\operatorname{im}(\tau)=\{\lambda \in \mathbb{R}: \exists i \in P$ with $\tau(i)=\lambda\}$. In this paper we use the operators $\wedge, \vee$ as the minimum and the maximum, respectively. Jiménez-Losada et al. (2010) introduced games with fuzzy communication structures. We consider $N$ our set of players.

Definition 1 A fuzzy communication structure over $N$ is $\gamma=(\tau, \rho)$ an undirected fuzzy graph over $N$, namely a fuzzy set of vertices $\tau \in[0,1]^{N}$ and a fuzzy set of links $\rho \in[0,1]^{L N}$ satisfying $\rho(i j) \leq \tau(i) \wedge \tau(j)$ for all $i j \in L N$. The set of fuzzy communication structures over $N$ is denoted by $\operatorname{FCS}^{N}$.

Hence, we will use fuzzy graph or fuzzy communication structure alike. We denote as $\gamma=0$ the null fuzzy graph where $\tau=0$ and $\rho=0$. Let $\gamma=(\tau, \rho) \in \operatorname{FCS}^{N}$ be a fuzzy communication structure. The set of vertices in $\gamma$ is $N(\gamma)=\operatorname{supp}(\tau)$ and the set of links is $L(\gamma)=\operatorname{supp}(\rho)$. We identify $\gamma$ with a real matrix $\gamma$ of size $n \times n$. We store the fuzzy set of vertices and the fuzzy set of links in a upper triangular matrix $\gamma$, where the elements in the diagonal are the levels of the vertices and the rest are the levels of the links.

$$
\gamma=\left[\begin{array}{cccc}
\gamma(11) & \gamma(12) & \cdots & \gamma(1 n) \\
0 & \gamma(22) & \cdots & \gamma(2 n) \\
\vdots & \vdots & \ddots & \vdots \\
0 & 0 & 0 & \gamma(n n)
\end{array}\right]
$$

For every link $i j$ of fuzzy graph $\gamma$, we have $\gamma(i j)=\rho(i j)$, otherwise $\gamma(i j)=0$. If a vertex is active $\gamma(i i)=\tau(i)$, otherwise $\gamma(i i)=0$. The number of non-zero elements of the matrix $\gamma$ is $|N(\gamma)|+|L(\gamma)|$.

Example 1 The fuzzy tree $\gamma$ and its matrix representation in Fig. 1.

$$
\gamma=\left[\begin{array}{cccc}
0.4 & 0.3 & 0.2 & 0 \\
0 & 0.6 & 0 & 0 \\
0 & 0 & 0.8 & 0.4 \\
0 & 0 & 0 & 0.5
\end{array}\right]
$$

All the communication structures over $N$ are fuzzy communication structures. But not only those, also the communication structures over $S$ for all coalition $S$. We set $C S_{0}^{N}=\bigcup_{\{S \subseteq N: S \neq \emptyset\}} C S^{S}$. These graphs are named crisp communication structures. 
Fig. 2 Crisp tree $g^{\gamma}$

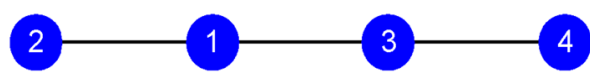

Every graph $g=(S, L) \in C S_{0}^{N}$ is identified with the fuzzy graph $\gamma=(\tau, \rho) \in$ $\operatorname{FCS}^{N}$, where $\tau=e^{S}$ and $\rho=e^{L}$.

In a fuzzy communication structure $\gamma=(\tau, \rho)$, the number $\tau(i)$ is interpreted as the real level of involvement of player $i \in N$ in a game situation and the number $\rho(i j)$ represents the maximal level to which the link $i j$ can be used. is

The crisp version of $\gamma$ is the graph $g^{\gamma}=(N(\gamma), L(\gamma))$. The minimal level in $\gamma$

$$
\wedge \gamma=\left(\bigwedge_{i \in N(\gamma)} \tau(i)\right) \wedge\left(\bigwedge_{i j \in L(\gamma)} \rho(i j)\right)
$$

Another fuzzy graph $\gamma^{\prime}=\left(\tau^{\prime}, \rho^{\prime}\right)$ over $N$ is a subgraph of $\gamma$ iff $\tau^{\prime} \leq \tau$ and $\rho^{\prime} \leq \rho$. We use in that case $\gamma^{\prime} \leq \gamma$. We defined three binary operations for fuzzy graphs in Jiménez-Losada et al. (2010). Let $\gamma=(\tau, \rho), \gamma^{\prime}=\left(\tau^{\prime}, \rho^{\prime}\right) \in \mathrm{FCS}^{N}$ be two fuzzy graphs over $N$ : (1) if $\tau(i)+\tau^{\prime}(i) \leq 1$ for all $i \in N$ then $\gamma+\gamma^{\prime}=\left(\tau+\tau^{\prime}, \rho+\rho^{\prime}\right)$, (2) if $\gamma^{\prime} \leq \gamma$ and $\rho(i j)-\rho^{\prime}(i j) \leq\left[\tau(i)-\tau^{\prime}(i)\right] \wedge\left[\tau(j)-\tau^{\prime}(j)\right]$ for all $i, j \in N$ then $\gamma-\gamma^{\prime}=\left(\tau-\tau^{\prime}, \rho-\rho^{\prime}\right)$, and (3) if $t \in[0,1]$ then $t \gamma=(t \tau, t \rho)$. The reader can see that the subtraction of fuzzy graphs is not always feasible as the opposite operation of the sum. The restriction of $\gamma$ to a coalition $S$ is $\gamma_{S}=\left(\tau_{S}, \rho_{S}\right) \leq \gamma$ verifying $\tau_{S}(i)=\tau(i) \wedge e_{i}^{S}$ for each player $i$ and $\rho_{S}(i j)=\rho(i j) \wedge e^{L(S)}(i j)$ for any link $i j$.

Also we can represent the crisp graph $g^{\gamma}$ corresponding to the graph $\gamma$ with a matrix $g^{\gamma}$ such that $g^{\gamma}(i j)=\lceil\gamma(i j)\rceil$ for $i, j \in N$. We use the notation

$$
N / \gamma=N(\gamma) / g^{\gamma}
$$

Example 2 The crisp tree $g^{\gamma}$, and its matrix representation, of the fuzzy graph in Example 1 (Fig. 2).

$$
g^{\gamma}=\left[\begin{array}{llll}
1 & 1 & 1 & 0 \\
0 & 1 & 0 & 0 \\
0 & 0 & 1 & 1 \\
0 & 0 & 0 & 1
\end{array}\right]
$$

Definition 2 A fuzzy communication game over $N$ is $(v, \gamma)$, where $v \in G^{N}$ and $\gamma \in \mathrm{FCS}^{N}$. The family of fuzzy communication games is $\mathrm{FCG}^{N}$ and $\mathrm{FCG}_{\mathrm{cf}}^{N}$ denotes those with cycle-free crisp version of the fuzzy communication structure.

Definition 3 A fuzzy communication value $F$ assigns to each fuzzy communication game $(v, \gamma) \in \mathrm{FCG}^{N}$ a payoff vector $F(v, \gamma) \in \mathbb{R}^{N}$. 
Aubin (1981) introduced partitions by levels to determine the worth of a fuzzy coalition in a given cooperative game. Jiménez-Losada et al., following Aubin and the Myerson model (Myerson 1977), defined in Jiménez-Losada et al. (2013) a way to get the total profit in a fuzzy communication structure for a game. They introduced the concept of partition by levels of a fuzzy graph as a finite sequence $\left(g_{k}, s_{k}\right)_{k=1}^{m}$ of pairs with $g_{k} \in C S_{0}^{N}$ and $s_{k} \in(0,1]$ which discards the information of the fuzzy communication structure, in the sense that $s_{k} g_{k} \leq \gamma-\sum_{l=1}^{k-1} s_{l} g_{l}$ for all $k>1$ and $\gamma-\sum_{k=1}^{m} s_{k} g_{k}=0$.

In this paper we focus on one of these partitions based in the Choquet integral (Choquet 1953). We consider that the object of the behavior of the players is the whole fuzzy graph, the set of vertices and links, as in the first model. So, the Choquet behavior says: players look first for the biggest crisp graph in the fuzzy communication structure and second for the uppest level to use all the elements of this graph. We can see again that there is only one partition representing this particular behavior of the players. Let $\gamma=(\tau, \rho) \in \mathrm{FCS}^{N}$ be a fuzzy communication structure. We construct this new partition with the following algorithm, named cg-partition.

Algorithm cg-partition $(\gamma)$

$$
k \leftarrow 0, c g \leftarrow \emptyset
$$

while $\gamma \neq 0$ do

$$
\begin{aligned}
& k \leftarrow k+1 \\
& s_{k} \leftarrow \wedge \gamma \\
& g_{k} \leftarrow g^{\gamma} \\
& c g \leftarrow c g \cup\left\{\left(g_{k}, s_{k}\right)\right\} \\
& \gamma \leftarrow \gamma-s_{k} g_{k}
\end{aligned}
$$

\section{end}

The partition by levels is $c g$.

Definition 4 Let $\gamma \in \mathrm{FCS}^{N}$. The Choquet by graphs partition by levels of $\gamma$ is the family $c g(\gamma)=\left(g_{k}, s_{k}\right)_{k=1}^{m}$ obtained by the $c g$-partition algorithm, where $g_{k} \in C S_{0}^{N}$ and $s_{k} \in[0,1]$ for all $k$. It is denoted as $c g(\gamma)$.

The $c g$-partition means that players try to get first the biggest graph and second the top level to connect it. The authors in Jiménez-Losada et al. (2013) defined a fuzzy version of the Myerson value for games using the $c g$-partition which is described by the Choquet formula

$$
M(v, \gamma)=\sum_{k=1}^{m} s_{k} \mu\left(v, g_{k}\right),
$$

where $c g(\gamma)=\left(g_{k}, s_{k}\right)_{k=1}^{m}$. Observe that the set of vertices of each $g_{k}$ is not always $N$, so if we denoted as $N_{k}$ the vertices of $g_{k}$ then $\mu\left(v, g_{k}\right)$ consists of applying the Myerson value on $C S^{N_{k}}$ and later extending the vector by zeros in $\mathbb{R}^{N}$. 
Fig. 3 Steps in the fuzzy graph of the example

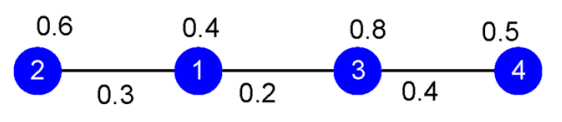

Step 1: After 0.2 units
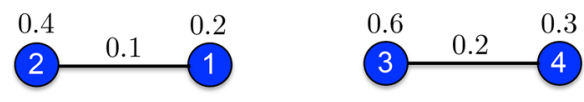

Step 2: After 0.1 units more

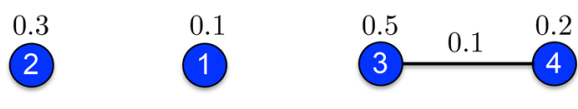

Step 3: After 0.1 units more

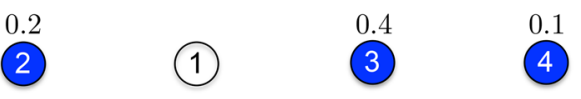

\section{Motivation}

Let us consider a situation in which a firm must supply energy to a set of four nodes each day. The nodes are able not only to accumulate energy but also to transmit energy to other nodes using the channels inside an existing network among them. We suppose known a maximal quantity of energy $q$ available for each day. Let $N=\{1,2,3,4\}$ be the set of the four nodes. For each subset of nodes $S$ we denote as $c(S)$ the fee of transmission of the $q$ units of energy to the network formed by the nodes in $S$. If the network is not connected then the cost must be the sum of the costs of its components. The problem is that each day neither all of the nodes need the same quantity of energy nor the channels are capable of conducing the same amount of energy. The conditions of the problem can be represented by a fuzzy communication structure for each day. To illustrate this idea let us suppose for a certain day the fuzzy graph $\gamma=(\tau, \rho)$ over $N$ in Fig. 1 with $\rho(1,3)=0.2, \rho(1,2)=0.3, \rho(3,4)=0.4 \tau(1)=0.4$, $\tau(2)=0.6, \tau(3)=0.8, \tau(4)=0.5$ and otherwise $\rho(i, j)=0$. The vertices represent the set of nodes (receivers, transmitters and accumulators of electricity) and the edges are transmitter of this energy. For instance, $c(\{1,2,3\})$ represents the saving of the following action: node 2 receives three units of energy, one is for it and the other two are sent by link 12 towards 1 , which catches one and transmits another through edge 13 towards node 3 , that receives which is left. It is evident that players must be connected.

The number over each node is the proportion of the $q$ units of energy that this node needs that day, and the number over every link is the transfer proportion of the $q$ units of energy. Hence we have a fuzzy communication game. To maximize its saving, the firm tries to use the minimal number of sources in the network. In fact when through a channel has passed the maximum amount of energy allowed, it is no longer available. This fact makes that the different nodes can stop being connected to the firm and it is obliged to introduce energy through several other nodes at the same 
time. We ask ourselves about the average maintenance saving of any node under these circumstances. If we look at the graph in Fig. 3, after transmitting 0.2 units of energy, edge 13 no longer exists. In order to be able to continue supplying energy, the firm must use a path which does not contain it, see Fig. 3. Nevertheless, there is no way that already communicates all nodes, so the firm must send the energy directly to a pair of nodes that are not connected. The next sending is 0.1 units worth. However, the saving can not be calculated by means of game $v$, so we assign to this situation a saving of $0.1 v(\{3,4\})+0.1 v(\{1,2\})$. Continuing with this action, the firm sees that nodes 1 and 2 are now isolated while 3 and 4 remain connected, so it must influence over three of the four nodes to continue providing energy to the nodes. Here the saving would be $0.1 v(\{1\})+0.1 v(\{2\})+0.1 v(\{3,4\})$. From now on, node 1 is supplied and passes individually to the other nodes the saving $0.2 v(\{2\}), 0.1 c(\{4\})$ and $0.4 v(\{3\})$. We suppose as saving game the characteristic function $v(S)=|S|^{2}$ for all $S \subseteq N$. A payoff vector of the fuzzy communication game constructed from the saving game allows us to obtain an allocation of the cost of the real use quantity among the nodes for each day. The fact that we take different nodes as sources and their rooted trees leads us to the philosophy of the AT value but adapted to fuzzy communication.

\section{The cg-average tree value}

We have to define a fuzzy version of the average tree value for cycle-free fuzzy communication games in the $c g$-way, we will named cg-average fuzzy value.

Definition 5 Let $(v, \gamma) \in \mathrm{FCG}^{N}$. For each coalition $S$ we denote $c g\left(\gamma_{S}\right)=$ $\left(g_{k}, s_{k}\right)_{k=1}^{m}$. The $c g$-restricted game $v^{\gamma} \in G^{N}$ is defined as

$$
v^{\gamma}(S)=\sum_{k=1}^{m} s_{k} v^{g_{k}}(S) .
$$

The $c g$-restricted game determines the worth of a coalition as the Myerson restricted game in a crisp communication structure but using the $c g$-partition of the fuzzy communication structure. Suppose $S \subseteq N$ with $c g\left(\gamma_{S}\right)=\left(g_{k}, s_{k}\right)_{k=1}^{m}$. Players in coalition $S$ look for the biggest communication structure among them $g_{1}$ at level $s_{1}$ and they obtain the worth $v^{g_{1}}(S)$. They have $\gamma-s_{1} g_{1}$, following the $c g$-algorithm, and they repeat the idea.

We follow now the process in Herings et al. (2008). A hierarchical fuzzy outcome associated with each player is defined on the class of fuzzy communication games such that the crisp version of the fuzzy graph is cycle free. If $\gamma \in \operatorname{FCS}_{\mathrm{cf}}^{N}$, then for each player $p \in N,\left(g^{\gamma}\right)^{p}$ is the crisp rooted tree at $p$ in the component of $\gamma$ containing $p$, we denote $\left(g^{\gamma}\right)^{p}$ as $\gamma^{p}$. The set of descendants of vertex $j$ on the rooted subtree $\left(g^{\gamma}\right)_{j}^{p}$ is denoted as $C^{\gamma^{p}}(j)$, and the set of children of the vertex $j$ on the rooted subtree $\left(g^{\gamma}\right)_{j}^{p}$ is denoted as $N^{\gamma^{p}}(j)$. 
Table 1 Matrices of the fuzzy graphs $\gamma_{k}$

$\left.\begin{array}{ccc}{\left[\begin{array}{cccc}0.4 & 0.3 & 0.2 & 0 \\ 0 & 0.6 & 0 & 0 \\ 0 & 0 & 0.8 & 0.4 \\ 0 & 0 & 0 & 0.5\end{array}\right]} & {\left[\begin{array}{cccc}0.2 & 0.1 & 0 & 0 \\ 0 & 0.4 & 0 & 0 \\ 0 & 0 & 0.6 & 0.2 \\ 0 & 0 & 0 & 0.3\end{array}\right]} \\ s_{2}=0.2 & =0.1\end{array}\right]$

Definition 6 Let $(v, \gamma) \in \mathrm{FCG}_{\mathrm{cf}}^{N}$. For each player $i \in N(\gamma)$, the component $j$ of the payoff vector associated with player $i$ ( $t^{i}$-payoff vector) is

$$
t_{j}^{i}(v, \gamma)=v^{\gamma}\left(C^{\gamma^{i}}(j)\right)-\sum_{u \in N^{\gamma^{i}}(j)} v^{\gamma}\left(C^{\gamma^{i}}(u)\right),
$$

for all $j \in N(\gamma)$. Notice that $C^{\gamma^{i}}(i)$ coincides with the component of $g^{\gamma}$ containing $i$.

Definition 7 Let $(v, \gamma) \in \mathrm{FCG}_{\mathrm{cf}}^{N}$, the $c g$-average tree value for a player $j$ is defined by

$$
A F_{j}(v, \gamma)=\frac{1}{\left|K_{j}\right|} \sum_{i \in K_{j}} t_{j}^{i}(v, \gamma)
$$

where $K_{j} \in N / \gamma$ and $j \in K_{j}$.

Example 3 Let $(v, \gamma) \in \mathrm{FCG}_{\mathrm{cf}}^{N}, v(S)=|S|^{2}$ for all $S \subseteq N$, and the fuzzy graph $\gamma$ of Fig. 1. To compute the characteristic function of the restricted game $v^{\gamma}$, it is necessary to obtain the $c g$-partition $\left(g_{k}, s_{k}\right)_{k=1}^{m}$, and the characteristic function of the restricted games $\left(v, g_{k}\right), k \in\{1, \ldots, m\}$.

Table 1 includes the matrices corresponding to the fuzzy graphs $\gamma_{k}=\gamma-s_{k} g_{k}$ in each step pf the $c g$-partition $\left(g_{k}, s_{k}\right)_{k=1}^{m}$, and Table 2 the crisp graphs $g_{k}$ and levels $s_{k}$. To compute number $t_{j}^{i}(v, \gamma)$, we consider $\gamma^{i}$. For example, we have $\gamma^{1}$ in Fig. 4. Table 3 includes the values of the restricted games $v^{g_{k}}$ and the restricted game $v^{\gamma}$ corresponding to the $c g$-partition $c g(\gamma)=\left(g_{k}, s_{k}\right)_{k=1}^{m}$.

Particularly to obtain $t_{j}^{1}(v, \gamma)$ for $j \in N(\gamma)$, first we calculate $C^{\gamma^{1}}(j)$ and $N^{\gamma^{1}}(j)$ (Table 4).

Replacing in the expression

$$
t_{j}^{1}(v, \gamma)=v^{\gamma}\left(C^{\gamma^{1}}(j)\right)-\sum_{u \in N^{\gamma 1}(j)} v^{\gamma}\left(C^{\gamma^{1}}(u)\right),
$$


Table 2 The $c g$-partition of $\gamma$

\begin{tabular}{|c|c|c|}
\hline $\begin{array}{c}{\left[\begin{array}{llll}1 & 1 & 1 & 0 \\
0 & 1 & 0 & 0 \\
0 & 0 & 1 & 1 \\
0 & 0 & 0 & 1\end{array}\right]} \\
s_{1}=0.2\end{array}$ & $\begin{array}{c}{\left[\begin{array}{llll}1 & 1 & 0 & 0 \\
0 & 1 & 0 & 0 \\
0 & 0 & 1 & 1 \\
0 & 0 & 0 & 1\end{array}\right]} \\
s_{2}=0.1\end{array}$ & $\begin{array}{c}{\left[\begin{array}{llll}1 & 0 & 0 & 0 \\
0 & 1 & 0 & 0 \\
0 & 0 & 1 & 1 \\
0 & 0 & 0 & 1\end{array}\right]} \\
s_{3}=0.1\end{array}$ \\
\hline $\begin{array}{c}{\left[\begin{array}{llll}0 & 0 & 0 & 0 \\
0 & 1 & 0 & 0 \\
0 & 0 & 1 & 0 \\
0 & 0 & 0 & 1\end{array}\right]} \\
s_{4}=0.1\end{array}$ & $\begin{array}{c}{\left[\begin{array}{llll}0 & 0 & 0 & 0 \\
0 & 1 & 0 & 0 \\
0 & 0 & 1 & 0 \\
0 & 0 & 0 & 0\end{array}\right]} \\
s_{5}=0.1\end{array}$ & $\begin{array}{c}{\left[\begin{array}{llll}0 & 0 & 0 & 0 \\
0 & 0 & 0 & 0 \\
0 & 0 & 1 & 0 \\
0 & 0 & 0 & 0\end{array}\right]} \\
s_{6}=0.2\end{array}$ \\
\hline
\end{tabular}

Matrices of the crisp graphs $g_{k}$ and levels $s_{k}$

Fig. 4 Rooted tree $\gamma^{1}$

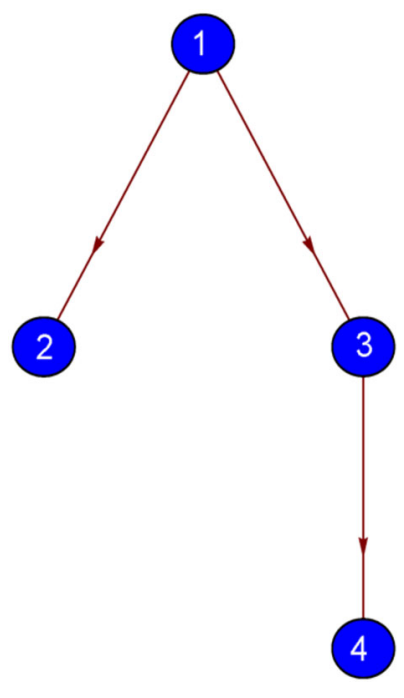

we have the $t^{1}$-payoff:

$$
\begin{aligned}
& t_{1}^{1}(v, \gamma)=v^{\gamma}(\{1,2,3,4\})-v^{\gamma}(\{2\})-v^{\gamma}(\{3,4\})=2.6 \\
& t_{2}^{1}(v, \gamma)=v^{\gamma}(\{2\})=0.6 \\
& t_{3}^{2}(v, \gamma)=v^{\gamma}(\{3,4\})-v^{\gamma}(\{4\})=1.6 \\
& t_{4}^{1}(v, \gamma)=v^{\gamma}(\{4\})=0.5
\end{aligned}
$$

Finally, $t^{1}(v, \gamma)=(2.6,0.6,1.6,0.5)$. Notice that this vector is efficient because the sum of its coordinates is $v^{\gamma}(N)$ (in this graph, we have only one component). The other vectors can be computed in the same way changing the hierarchy. Following the same process, for the rest of the vertices, we obtain the $t^{i}$-payoffs

$$
\begin{aligned}
& t^{1}(v, \gamma)=(2.6,0.6,1.6,0.5) \mid t^{2}(v, \gamma)=(1.2,2,1.6,0.5) \\
& t^{3}(v, \gamma)=(1,0.6,3.2,0.5) \quad \mid t^{4}(v, \gamma)=(1,0.6,1.6,2.1)
\end{aligned}
$$


Table 3 Determining the restricted game $v^{\gamma}$

\begin{tabular}{llllllll}
\hline$S$ & $s_{1} v^{g_{1}}$ & $s_{2} v^{g_{2}}$ & $s_{3} v^{g_{3}}$ & $s_{4} v^{g_{4}}$ & $s_{5} v^{g_{5}}$ & $s_{6} v^{g_{6}}$ & $v^{\gamma}$ \\
\hline$\emptyset$ & 0. & 0. & 0. & 0. & 0. & 0. & 0. \\
$\{1\}$ & 0.2 & 0.1 & 0.1 & 0. & 0. & 0. & 0.4 \\
$\{2\}$ & 0.2 & 0.1 & 0.1 & 0.1 & 0.1 & 0. & 0.6 \\
$\{3\}$ & 0.2 & 0.1 & 0.1 & 0.1 & 0.1 & 0.2 & 0.8 \\
$\{4\}$ & 0.2 & 0.1 & 0.1 & 0.1 & 0. & 0. & 0.5 \\
$\{1,2\}$ & 0.8 & 0.4 & 0.2 & 0.1 & 0.1 & 0. & 1.6 \\
$\{1,3\}$ & 0.8 & 0.2 & 0.2 & 0.1 & 0.1 & 0.2 & 1.6 \\
$\{1,4\}$ & 0.4 & 0.2 & 0.2 & 0.1 & 0. & 0. & 0.9 \\
$\{2,3\}$ & 0.4 & 0.2 & 0.2 & 0.2 & 0.2 & 0.2 & 1.4 \\
$\{2,4\}$ & 0.4 & 0.2 & 0.2 & 0.2 & 0.1 & 0. & 1.1 \\
$\{3,4\}$ & 0.8 & 0.4 & 0.4 & 0.2 & 0.1 & 0.2 & 2.1 \\
$\{1,2,3\}$ & 1.8 & 0.5 & 0.3 & 0.2 & 0.2 & 0.2 & 3.2 \\
$\{1,2,4\}$ & 1. & 0.5 & 0.3 & 0.2 & 0.1 & 0. & 2.1 \\
$\{1,3,4\}$ & 1.8 & 0.5 & 0.5 & 0.2 & 0.1 & 0.2 & 3.3 \\
$\{2,3,4\}$ & 1. & 0.5 & 0.5 & 0.3 & 0.2 & 0.2 & 2.7 \\
$\{1,2,3,4\}$ & 3.2 & 0.8 & 0.6 & 0.3 & 0.2 & 0.2 & 5.3 \\
\hline
\end{tabular}

Table 4 Sets $C^{\gamma^{1}}(j)$ and $N \gamma^{1}(j)$

\begin{tabular}{lllll}
\hline$j$ & 1 & 2 & 3 & 4 \\
\hline$C^{\gamma^{1}}(j)$ & $\{1,2,3,4\}$ & $\{2\}$ & $\{3,4\}$ & $\{4\}$ \\
$N^{\gamma^{1}(j)}$ & $\{2,3\}$ & $\emptyset$ & $\{4\}$ & $\emptyset$ \\
\hline
\end{tabular}

In this example, $\gamma$ is connected. Also $N(\gamma)=N$.

$$
\begin{aligned}
A F(v, \gamma) & =\frac{1}{|N|} \sum_{i \in N} t^{i}(v, \gamma)=\frac{1}{4}\left(t^{1}(v, \gamma)+t^{2}(v, \gamma)+t^{3}(v, \gamma)+t^{4}(v, \gamma)\right. \\
& =(1.45,0.95,2.0,0.9)
\end{aligned}
$$

Remark 1 Following Jiménez-Losada et al. (2013) a natural formula for the $c g$-average tree value would be a Choquet expression as

$$
F(v, \gamma)=\sum_{k=1}^{m} s_{k} A T\left(v, g_{k}\right)
$$

for all $(v, \gamma) \in \mathrm{FCG}_{\mathrm{cf}}^{N}$ where $c g(\gamma)=\left(g_{k}, s_{k}\right)_{k=1}^{m}$. The average tree value $A T$ is evaluated in the game restricted to the vertices of each $g_{k}$ and then extending by zeros. But the next example shows that this formula is not valid for our value. In general $F(v, \gamma) \neq A F(v, \gamma)$. Since Example 3, the average tree values for graphs $g_{k}$ corresponding to $\operatorname{cg}(\gamma)$ are: 


$$
\begin{aligned}
& A T\left(v, g_{1}\right)=(5.5,2.5,5.5,2.5) \mid A T\left(v, g_{2}\right)=(2,2,2,2) \\
& A T\left(v, g_{3}\right)=(1,1,2,2) \\
& A T\left(v, g_{5}\right)=(0,1,1,0) \\
& A T\left(v, g_{4}\right)=(0,1,1,1) \\
& F(v, \gamma)=(1.4,1,1.9,1) \neq(1.45,0.95,2,0.9)=A F(v, \gamma) \text {. }
\end{aligned}
$$

\section{Axiomatization of the $c g$-average tree value}

Now, we look for an axiomatization of the $c g$-average tree value following Herings et al. (2008). The set of cycle-free fuzzy communication structures over $N$ is denoted by $\mathrm{FCS}_{\mathrm{cf}}^{N}$. Consider the following axioms for a given fuzzy communication value $F$ defined over $\mathrm{FCG}_{\mathrm{cf}}^{N}$.

We are interesting in obtaining an allocation of the saving for each structure, namely we need an efficient payoff vector for the worth of the great coalition in the $c g$-restricted game.

Fuzzy efficiency by components For all $(v, \gamma) \in \mathrm{FCG}_{\mathrm{cf}}^{N}$, if $K \in N / \gamma$ then by components

$$
\sum_{i \in K} F_{i}(v, \gamma)=v^{\gamma}(K)
$$

The subgraph $\gamma_{-i j}^{t}=\left(\tau_{-i j}^{t}, \rho_{-i j}^{t}\right)$ represents the fuzzy graph $\gamma$ modified by reducing to $t \in[0, \rho(i j)]$ the capacity of $i j \in L(\gamma)$, that is, $\tau_{-i j}^{t}=\tau$ and

$$
\rho_{-i j}^{t}\left(i^{\prime} j^{\prime}\right)= \begin{cases}\rho\left(i^{\prime} j^{\prime}\right), & \text { if } i^{\prime} j^{\prime} \neq i j \\ t, & \text { if } i^{\prime} j^{\prime}=i j\end{cases}
$$

Fuzzy component fairness For any link $i j \in \gamma$ with $\rho(i j)>0$ and $t \in[0, \rho(i j)]$ it holds that

$$
\frac{1}{\left|K_{i}^{i j}\right|} \sum_{h \in K_{i}^{i j}}\left[F_{h}(v, \gamma)-F_{h}\left(v, \gamma_{-i j}^{t}\right)\right]=\frac{1}{\left|K_{j}^{i j}\right|} \sum_{r \in K_{j}^{i j}}\left[F_{r}(v, \gamma)-F_{r}\left(v, \gamma_{-i j}^{t}\right)\right] .
$$

This axiom means that when we reduce to $t$ the level for a link $i j \in \gamma$, the resulting average change for payoff of the players in $K_{i}^{i j}$ is equal to the average change for payoff to the players in $K_{j}^{i j}$. Notice that when we reduce to $0<t<\rho(i j)$, the axiom is satisfied trivially to the playes in $K_{j}^{i j}$.

Lemma 1 Let $(v, \gamma) \in \mathrm{FCG}_{\mathrm{cf}}^{N}$, with $\gamma$ connected. For any players $i, j \in N$ the $t^{i}$ payoff vector satisfies

$$
\sum_{h \in C^{\gamma^{i}}(j)} t_{h}^{i}(v, \gamma)=v^{\gamma}\left(C^{\gamma^{i}}(j)\right)
$$


Proof We prove the result by induction in the height $H$ of vertex $j$ in the rooted tree $\gamma^{i}$. If $H=0$, vertex $j$ is a leaf, then the result is obvious by definition. Suppose that the result is true for any vertex with height $H-1, H>1$, and we prove the result for a vertex $j$ with height $H$. We have that

$$
\begin{aligned}
\sum_{h \in C^{\gamma^{i}}(j)} t_{h}^{i}(v, \gamma) & =t_{j}^{i}(v, \gamma)+\sum_{u \in N^{\gamma^{i}}(j)} \sum_{h \in C^{\gamma^{i}}(u)} t_{h}^{i}(v, \gamma) \\
& =t_{j}^{i}(v, \gamma)+\sum_{u \in N^{\gamma^{i}}(j)} v^{\gamma}\left(C^{\gamma^{i}}(u)\right)=v^{\gamma}\left(C^{\gamma^{i}}(j)\right)
\end{aligned}
$$

The $t^{i}$-payoff of player $j$ is equal to the contribution of player $j$ when he joins his descendants in the subtree $\left(g^{\gamma}\right)_{j}^{i}$. When the set $C^{\gamma^{i}}(j)$ is connected, player $j$ connects all the subsets of descendants of his children into one connected set and receives his marginal contribution to it.

Theorem 1 The cg-average tree value satisfies fuzzy efficiency by components and fuzzy components fairness.

Proof Let $v \in G^{N}$. We take $(v, \gamma) \in \mathrm{FCG}_{\mathrm{cf}}^{N}$ with $c g$-partition $c g(\gamma)=\left(g_{k}, s_{k}\right)_{k=1}^{m}$. Let $K \in N / \gamma$. As each player $i \in K$ is top player in the tree $\gamma^{i}$, the rooted tree associates with $i$, then $C^{\gamma}(i)=K$. Applying the above lemma, as $C^{\gamma^{i}}(i)=K$, we obtain

$$
\begin{aligned}
\sum_{h \in K} A F_{h}(v, \gamma) & =\frac{1}{|K|} \sum_{h \in K} \sum_{i \in K} t_{h}^{i}(v, \gamma)=\frac{1}{|K|} \sum_{i \in K} \sum_{h \in K} t_{h}^{i}(v, \gamma) \\
& =\frac{1}{|K|} \sum_{i \in K} \sum_{h \in C \gamma^{i}(i)} t_{h}^{i}(v, \gamma)=\frac{1}{|K|} \sum_{i \in K} v^{\gamma}\left(C^{\gamma^{i}}(h)\right)=v^{\gamma}(K) .
\end{aligned}
$$

Now, we check fuzzy component fairness for $t=\rho(i j)$. Players $i, j$ are in different connected components in $\gamma_{-i j}^{\rho(i j)}$, noted, respectively, by $K_{i}^{i j}$ and $K_{j}^{i j}$. Fuzzy efficiency by components implies that

$$
\sum_{h \in K_{i}^{i j}} A F_{h}\left(v, \gamma_{-i j}^{t}\right)=v^{\gamma}\left(K_{i}^{i j}\right) \text { and } \sum_{h \in K_{j}^{i j}} A F_{h}\left(v, \gamma_{-i j}^{t}\right)=v^{\gamma}\left(K_{j}^{i j}\right)
$$

Observe that for each $h^{\prime} \in K_{j}^{i j}$ we have $C^{\gamma^{h^{\prime}}}(i)=K_{j}^{i j}$, so by Lemma 1 it holds in $\gamma$

$$
\sum_{h \in K_{j}^{i j}} t_{h}^{h^{\prime}}(v, \gamma)=v^{\gamma}\left(K_{j}^{i j}\right)
$$


On the other hand, for $h^{\prime} \in K_{i}^{i j}$ we get $C^{\gamma^{h^{\prime}}}(j)=K_{j}^{i j}$ and $C^{\gamma^{h^{\prime}}}\left(h^{\prime}\right)=K$. Hence Lemma 1 again implies

$$
\begin{aligned}
\sum_{h \in K_{i}^{i j}} t_{h}^{h^{\prime}}(v, \gamma) & =\sum_{h \in K} t_{h}^{h^{\prime}}(v, \gamma)-\sum_{h \in K_{j}^{i j}} t_{h}^{h^{\prime}}(v, \gamma) \\
& =v^{\gamma}(K)-v^{\gamma}\left(K_{j}^{i j}\right) .
\end{aligned}
$$

Next we get using (2) and (3),

$$
\begin{aligned}
\sum_{h \in K_{i}^{i j}} A F_{h}(v, \gamma) & =\sum_{h \in K_{i}^{i j}} \frac{1}{|K|} \sum_{h^{\prime} \in K} t_{h}^{h^{\prime}}(v, \gamma) \\
& =\sum_{h \in K_{i}^{i j}} \frac{1}{|K|}\left[\sum_{h^{\prime} \in K_{i}^{i j}} t_{h}^{h^{\prime}}(v, \gamma)+\sum_{h^{\prime} \in K_{j}^{i j}} t_{h}^{h^{\prime}}(v, \gamma)\right] \\
& =\frac{\left|K_{i}^{i j}\right|\left[v^{\gamma}(K)-v^{\gamma}\left(K_{j}^{i j}\right)\right]+\left|K_{j}^{i j}\right| v^{\gamma}\left(K_{i}^{i j}\right)}{|K|} .
\end{aligned}
$$

Finally since (1),

$$
\begin{aligned}
\sum_{h \in K_{i}^{i j}} A F_{h}(v, \gamma) & =v^{\gamma}\left(K_{i}^{i j}\right)+\frac{\left|K_{i}^{i j}\right|}{|K|}\left[v^{\gamma}(K)-v^{\gamma}\left(K_{j}^{i j}\right)-v^{\gamma}\left(K_{i}^{i j}\right)\right] \\
& =\sum_{h \in K_{i}^{i j}} A F_{h}\left(v, \gamma_{-i j}^{t}\right)+\frac{\left|K_{i}^{i j}\right|}{|K|}\left[v^{\gamma}(K)-v^{\gamma}\left(K_{j}^{i j}\right)-v^{\gamma}\left(K_{i}^{i j}\right)\right] .
\end{aligned}
$$

So,

$$
\sum_{h \in K_{i}^{i j}}\left[A F_{h}(v, \gamma)-A F_{h}\left(v, \gamma_{-i j}^{t}\right)\right]=\frac{\left|K_{i}^{i j}\right|}{|K|}\left[v^{\gamma}(K)-v^{\gamma}\left(K_{j}^{i j}\right)-v^{\gamma}\left(K_{i}^{i j}\right)\right]
$$

If we swap $i$ and $j$ in the above reasoning we obtain the result,

$$
\sum_{h \in K_{j}^{i j}}\left[A F_{h}(v, \gamma)-A F_{h}\left(v, \gamma_{-i j}^{t}\right)\right]=\frac{\left|K_{j}^{i j}\right|}{|K|}\left[v^{\gamma}(K)-v^{\gamma}\left(K_{j}^{i j}\right)-v^{\gamma}\left(K_{i}^{i j}\right)\right]
$$

If $t<\rho(i j)$ then $K_{i}^{i j}=K_{i j}^{j}=K$, therefore the axiom is true.

Theorem 2 says that our fuzzy communication value is the only one satisfying these two axioms. We follow the proof of the crisp case in Herings et al. (2008). 
Theorem 2 The cg-average tree value is the only cycle-free fuzzy communication value satisfying fuzzy efficiency by components and fuzzy component fairness.

Proof Consider $F$ a fuzzy communication value satisfying these axioms. Our proof will use the same reasoning in each connected component $K$ of $\gamma$. As $\gamma_{K}$ is connected we have $|K|-1$ links in $L\left(\gamma_{K}\right)$. Fuzzy efficiency by components implies for each link $i j \in L(\gamma)$ two equations as in (1),

$$
\sum_{h \in K_{i}^{i j}} F_{h}\left(v, \gamma_{-i j}^{\rho(i j)}\right)=v^{\gamma}\left(K_{i}^{i j}\right) \text { and } \sum_{h \in K_{j}^{i j}} F_{h}\left(v, \gamma_{-i j}^{\rho(i j)}\right)=v^{\gamma}\left(K_{j}^{i j}\right)
$$

where $K_{i}^{i j}, K_{j}^{i j}$ are again the connected components containing $i, j$ in $\gamma_{-i j}^{\rho(i j)}$. From these two equations and using fuzzy component fairness, we obtain this other one,

$$
\begin{aligned}
& \left|K_{j}^{i j}\right| \sum_{h \in K_{i}^{i j}} F_{h}(v, \gamma)-\left|K_{i}^{i j}\right| \sum_{h \in K_{j}^{i j}} F_{h}(v, \gamma) \\
& =\left|K_{j}^{i j}\right| \sum_{h \in K_{i}^{i j}} F_{h}\left(v, \gamma_{-i j}^{\rho(i j)}\right)-\left|K_{i}^{i j}\right| \sum_{h \in K_{j}^{i j}} F_{h}\left(v, \gamma_{-i j}^{\rho(i j)}\right) \\
& =\left|K_{j}^{i j}\right| v^{\gamma}\left(K_{i}^{i j}\right)-\left|K_{i}^{i j}\right| v^{\gamma}\left(K_{j}^{i j}\right)=R_{i j} .
\end{aligned}
$$

Adding efficiency by components for $(v, \gamma)$ and component $K$ we get a linear equation system with size $|K| \times|K|$,

$$
\left\{\begin{array}{l}
\left|K_{j}^{i j}\right| \sum_{h \in K_{i}^{i j}} F_{h}(v, \gamma)-\left|K_{i}^{i j}\right| \sum_{h \in K_{j}^{i j}} F_{h}(v, \gamma)=R_{i j} \quad \forall i j \in L\left(\gamma_{K}\right) \\
\sum_{h \in K} F_{h}(v, \gamma)=v^{\gamma}(K) .
\end{array}\right.
$$

This system is determinate compatible, as we see analyzing its matrix of coefficients. Take any vertex $p \in K$ and suppose any total order $<_{p}$ of the players in $K$ such that $i \notin C^{\gamma_{K}^{p}}(j)$ if $i<_{p} j$. We sort the columns of the matrix by this order. Next we sort the rows of the matrix. We put the last equation in the first row of the matrix, denote $r$. Each link $i j$ is taken with $i<_{p} j$ and its equation is put in the position of $j$ in the order $<_{p}$, we denote this row as $r(j)$. Observe that the elements in $r(j)$ are $\left\{\left|K_{j}^{i j}\right|, \ldots,\left|K_{j}^{i j}\right|,-\left|K_{i}^{i j}\right|\right\}$ till column $j$ since the chosen order. We do $r(j)-\left|K_{j}^{i j}\right| r$ for all $j \neq p$, transforming our matrix in an upper superior matriz with main diagonal $\{1,-|K|, \ldots,-|K|\}$.

Now we show a property of stability of the $c g$-average tree value and as a consequence give an alternative axiomatization based on this property. First we recall the notion of Core for the game $v^{\gamma}$ under the communication structure $\gamma$.

$$
\operatorname{Core}(v, \gamma)=\left\{x \in \mathbb{R}^{N}: v^{\gamma}(S) \leqslant x(S) \forall S, v^{\gamma}(N)=x(N)\right\},
$$


where $x(S)=\sum_{i \in S} x_{i}$. Let $\pi$ be a permutation of $N$, the corresponding marginal vector $m^{\pi}(v, \gamma) \in \mathbb{R}^{N}$ assigns to every player $i$ a payoff $m_{i}^{\pi}(v, \gamma)=v^{\gamma}\left(\pi^{i} \cup i\right)-$ $v^{\gamma}\left(\pi^{i}\right)$, where $\pi^{i}$ is the set of players preceding $i$, the lowers of $i$, in the permutation $\pi$. Notice that for a hierarchical cycle-free fuzzy communication structure, with $i$ as top player, and taking $(\pi, i)$ as the order determined by the hierarchy, we have $t_{j}^{i}\left(v^{\gamma}, \gamma\right)=$ $m_{j}^{(\pi, i)}(v, \gamma)$. For example, the permutation associated to the order $4<1<3<2$ is $\pi=(2314)$. For $j=3$,

$$
m_{3}^{\pi}(v, \gamma)=v^{\gamma}(\{1,4\} \cup\{3\})-v^{\gamma}(\{1,4\}) .
$$

Notice that this component is the same as the third component of $t^{2}(v, \gamma)$.

Theorem 3 The cg-average tree value is the only cycle-free fuzzy communication value satisfying stability and fuzzy component fairness.

Proof A game $v$ is superadditive if for all $S, T \in 2^{N}$ with, $S \cap T=\emptyset, v(S \cup T) \geqslant$ $v(S)+v(T)$. In Demange (2004), the authors show that the hierarchical outcomes $t^{i}$ are in the core of superadditive games. Jiménez-Losada et al. (2010) show that the game $v^{\gamma}$ is superadditive for any game $v$. The core is convex and contains the marginal vectors $m_{j}^{(\pi, i)}(v, \gamma)$. On the other hand $t_{j}^{i}(v, \gamma)=m_{j}^{(\pi, i)}(v, \gamma)$, where $m_{j}^{(\pi, i)}(v, \gamma)$ is the marginal vector $m_{j}^{(\pi, i)}(v, \gamma)$ when the top player is $i$. This implies that $t^{i}(v, \gamma) \in$ Core $(v, \gamma)$ and as $A F(v, \gamma)$ is the average of these vectors, we have that $A F(v, \gamma)$ is also on the $\operatorname{Core}(v, \gamma)$.

Obviously, as all the payoff vectors in $\operatorname{Core}(v, \gamma)$ satisfy efficiency, then since the above theorem there is only one value satisfying the axioms in the statement.

\section{Computing the $c g$-average tree value}

In this section, we describe the algorithm $A F$ value to compute the $c g$-average tree value, and we study the complexity of it. Let $B$ be an algorithm. The time complexity of $B$ is measured by a function $f: \mathbb{Z}_{+} \rightarrow \mathbb{Z}_{+}$where $f(n)$ is the maximal number of iterations in a universal Turing machine (before halting) in relation to the size of the input $n$. Let $f, g: \mathbb{Z}_{+} \rightarrow \mathbb{Z}_{+}$. Following $\mathcal{O} \Omega \Theta$-notation, proposed by Knuth (1976), we use $f=\mathcal{O}(g)$ if there are $c, n_{0} \in \mathbb{Z}_{+}$such that $f(n) \leq c g(n)$ for all $n \geq n_{0}$. In that case, we say $f$ is of the order of $g$.

Let $(v, \gamma) \in \mathrm{FCG}_{\mathrm{cf}}^{N}$. We explain the process to compute the $c g$-average tree value with a tree, namely $g^{\gamma}$ is a tree. We describe the algorithm $A F$ value in six steps.

Step 1. Introduction of fuzzy graph $\gamma$ and game $(N, v)$.

Step 2. The Choquet by graphs partition, with $\gamma=(\tau, \rho)$, where $g^{\gamma}$ is a tree.

$$
\operatorname{cg}(\gamma)=\left(g_{k}, s_{k}\right)_{k=1}^{m}
$$

Step 3. The characteristic function of the restricted games $v^{g_{k}}, k=1, \ldots, m$.

$$
v^{g_{k}}(S)=\sum_{T \in S / g_{k}} v(T)
$$


Step 4. The characteristic function of the $c g$-restricted fuzzy game $v^{\gamma}$.

$$
v^{\gamma}(S)=\sum_{k=1}^{m} s_{k} v^{g_{k}}(S)
$$

Step 5. The number $t_{j}^{i}(v, \gamma)$ for every rooted subtree $g_{j}^{i}$, with $i, j=1, \ldots, n$.

$$
t_{j}^{i}(v, \gamma)=v^{\gamma}\left(C^{\gamma^{i}}(j)\right)-\sum_{u \in N^{\gamma^{i}}(j)} v^{\gamma}\left(C^{\gamma^{i}}(u)\right)
$$

Step 6. The $c g$-average tree value with the formula,

$$
A F_{j}(v, \gamma)=\frac{1}{|N|} \sum_{i \in N} t_{j}^{i}(v, \gamma) \text { for } j \in\{1, \ldots, n\}
$$

cg-partition algorithm

We construct the Choquet by graphs partition $c g(\gamma)=\left(g_{k}, s_{k}\right)_{k=1}^{m}$ with the $c g$ partition algorithm. We use the matrix representation of $\gamma$, to obtain the fuzzy graphs $\gamma_{k}$ and the crisp graphs $g_{k}$. The time complexity of cg-partition algorithm is $\mathcal{O}\left(\mathrm{m}^{2}\right)$ (Gallego et al. 2014), where $m=|N(\gamma)|+|L(\gamma)|$. For a fuzzy tree, $|N(\gamma)| \leq$ $n,|L(\gamma)| \leq n-1$, hence is required a time $\mathcal{O}\left(n^{2}\right)$.

Restricted game algorithm

To calculate the $c g$-average tree value, for $(v, \gamma) \in \mathrm{FCG}_{\mathrm{cf}}^{N}$, also it is necessary to determine first the characteristic function of the $c g$-restricted game $v^{\gamma}$ as

$$
v^{\gamma}(S)=\sum_{k=1}^{m} s_{k} v^{g_{k}}(S)
$$

We use the algorithm restricted game to compute the characteristic function of the restricted games $\left(v, g_{k}\right) \in C G^{N}, k=1, \ldots, m$. The computational cost of this algorithm is very high, since to compute the games $v^{g_{k}}$ it is necessary, for every coalition, previously to obtain the set of all the connected components in the induced subgraph by coalition $S$ in the graph $g_{k}$.

$$
v^{g_{k}}(S)=\sum_{R \in S / g_{k}} v(R)
$$

Let $(v, g) \in C G^{N}$ be, to compute the characteristic function $v^{g}$, we use the algorithm restricted game. 
Algorithm restricted game $(v, g)$

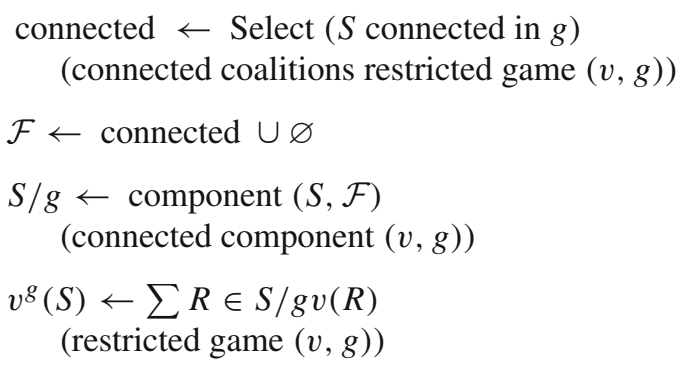

end

We need to calculate the set $\mathcal{F}^{g}=\left\{S \in 2^{N}: g_{S}\right.$ is connected $\}$ and $S / g$, where $g$ is tree. To calculate the feasible coalitions in $\mathcal{F}^{g}$, we can determine if a graph is connected or not by a depth first search (DFS) (Cormen et al. 2001) we needs a time $\mathcal{O}(|N(g)|+|L(g)|)=\mathcal{O}(n+n-1)=\mathcal{O}(n)$.

The worst case is a star tree (a tree with a central node, $n-1$ children, and height 1), in this case $\left|\mathcal{F}^{g}\right|=n+2^{n-1}$, if $S \in \mathcal{F}^{g} S / g=\{S\}$, if $S \notin \mathcal{F}^{g}$ it is necessary to do $|S|$ ! comparisons to determine the set $S / g$, the are $\left(\begin{array}{c}n-1 \\ i\end{array}\right)$ coalitions of size $i$,; therefore, it is necessary

$$
\begin{aligned}
\sum_{i=2}^{n-1}\left(\begin{array}{c}
n-1 \\
i
\end{array}\right) i ! & =-n+e \Gamma(n, 1)<e \Gamma(n) \\
& =e(n-1) ! \in \mathcal{O}((n-1) !) .
\end{aligned}
$$

Hence, we can to compute $v^{g}$ in a time

$$
\mathcal{O}\left(\max \left(n, 2^{n-1},(n-1) !\right)\right)=\mathcal{O}((n-1) !) .
$$

We suppose known games $v^{g_{k}}(S), k=1, \ldots, m$, as $m \leq|\operatorname{im}(\tau) \cup \operatorname{im}(\rho)| \leq$ $n+n-1=2 n-1$, we can compute $v^{\gamma}$ in a time $\mathcal{O}(\max (n,(n-1) !)=\mathcal{O}((n-1) !)$. tvalue algorithm

To compute the numbers $t_{j}^{i}(v, \gamma)$, for all $i, j \in N(\gamma)$, we will use the algorithm tvalue based on the (DFS) algorithm. Fixed the rooted tree $g^{\gamma^{i}}$, we locate $N^{\gamma^{i}}(i)$ and store them in a list, and then delete the node $i$. Next, we consider the directed routed subtrees $g_{j}^{\gamma^{i}}$ for all $j \in N^{\gamma^{i}}(i)$ corresponding to the stored nodes and repeat this process in a lexicographic order. We will continue the process until we visit all the nodes of the tree $g^{\gamma^{i}}$, simultaneously we are storing the visited nodes in a list, following an order in which they have been considered as roots of the sub-trees $g_{j}^{\gamma^{i}}$ in this process; in another list we store the unvisited nodes, before the beginning of each step. We obtain, for each step, $\left\{C^{\gamma^{i}}(u): u \in N^{\gamma^{i}}(j)\right\}$. Finally, we calculate the numbers $t_{j}^{i}(v, \gamma)$ and store them in a table $t(v, \gamma)_{n \times n}$. AF-value algorithm

To compute the $c g$-average tree value, we use the algorithm $A F$ value. 
Algorithm $A F$-value $(v, \gamma)$

cg $\leftarrow$ cg-partition $(\gamma)$

$\left(\operatorname{cg}\right.$-partition $\left.\operatorname{cg}(\gamma)=\left(s_{k}, g_{k}\right)_{k=1}^{k=m}\right)$

for $k=1$ to $m$

$v^{g_{k}} \leftarrow$ restricted game $(v, g)$

$v^{\gamma}(S) \leftarrow \sum_{k=1}^{m} s_{k} v^{g_{k}}(S)$

(cg-restricted game $(v, \gamma))$

end

$t(v, \gamma) \leftarrow$ tvalues $(\gamma)$

for $\mathbf{i} \in|N(\gamma)|$

$A F(v, \gamma) \leftarrow \frac{1}{|N|} \sum_{i \in N} t^{i}(v, \gamma)$

$(c g$-average tree value $(v, \gamma))$

end

Knowing $t(v, \gamma)$ we can compute $A F(v, \gamma)$ in linear time $\mathcal{O}(n)$.

Therefore, considering the six steps, the computational complexity of AF-value algorithm is

$$
\mathcal{O}\left(\max \left(n, 2^{n},(n-1) !, m, n, n\right)=\mathcal{O}((n-1) !) .\right.
$$

We studied in Jiménez-Losada et al. (2013) the complexity of the $c g$-Myerson value, for a tree is $\mathcal{O}\left(n 3^{n}\right)$. Thus the calculus of the $c g$-average tree value has greater complexity than the Myerson value (from 12 players). This issue occurs because we cannot use a Choquet formula (see Remark 1) as in the $c g$-Myerson value (see formula below Definition 4).

Example 4 Let $(v, \gamma) \in \mathrm{FCG}_{\mathrm{cf}}^{N}$ be, $v(S)=|S|^{2}$ for all $S \subseteq N$, and the fuzzy graph $\gamma$ of Fig. 5, the $c g$-average tree value, obtained by the $A F$-value algorithm is in Table 5.

\section{Conclusions}

In games with cycle-free communication structures the average tree value is an interesting solution for two reasons, it is an easy solution for calculating and also it is always stable. In this article we have developed a new value, the $c g$-average tree value for games with fuzzy cycle-free communication situations with similar good conditions. We have provided with an axiomatization and a property of stability. Also, we have studied the computational complexity of the AF-value algorithm and illustrated the calculation process. We also introduce an application of the value where this solution is reasonable. The stability in cooperative games is given by the concept of core and this one is defined by the idea of domination. Hence, a future work is to extend this mechanism to interval-valued fuzzy graphs following Debnath (2013). 
Fig. 5 Fuzzy graph $\gamma$

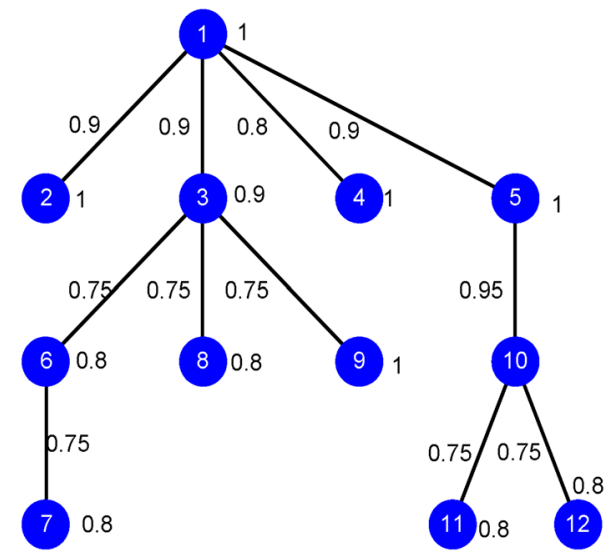

Table 5 The $c g$-average tree value and the $c g$-Myerson value

\begin{tabular}{lccccc}
\hline Player & $A F(v, \gamma)$ & $M(v, \gamma)$ & Player & $A F(v, \gamma)$ & $M(v, \gamma)$ \\
\hline 1 & 37.21670 & 21.57860 & 2 & 2.48333 & 6.10833 \\
3 & 29.16670 & 18.91190 & 4 & 2.31667 & 5.72500 \\
5 & 12.09170 & 14.53690 & 6 & 5.925 & 8.92857 \\
7 & 2.17500 & 4.75357 & 8 & 2.17500 & 5.35000 \\
9 & 2.17500 & 5.35000 & 10 & 13.22500 & 12.47860 \\
11 & 2.17500 & 4.78929 & 12 & 2.17500 & 4.78929 \\
\hline
\end{tabular}

Acknowledgements This research has been supported by the Spanish Ministry of Education an Science, under Grant MTM2017-83455-P, and by the FQM237 Grant of the Andalusian Government.

\section{References}

Aubin JP (1981) Cooperative fuzzy games. Math Oper Res 6:1-13

Bilbao JM, Fernández JR, López JJ (2002) On the complexity of computing values of restricted games. Int J Found Comput Sci 13:633-651

Choquet C (1953) Theory of capacities. Ann Inst Fourier Grenoble 5:131-295

Contreras J (1997) A cooperative game theory approach to transmission planning in power systems. Ph.D. Thesis. University of California, Berkeley

Cormen TH, Leierson CE, Rivest RL, Stein C (2001) Introduction to algorithms, 2nd edn. The MIT Press, Institute of Technology, Cambridge

Debnath P (2013) Domination in interval-valued fuzzy graphs. Ann Fuzzy Math Inform 6:363-370

Demange G (2004) On group stability in hierarchies and networks. J Political Econ 112:754-778

Driessen TSH (1988) Cooperative games, solutions and applications. Theory and decision library. Springer, Berlin

Fernández JR, Algaba E, Bilbao JM, Jimé nez A, Jiménez N, López JJ (2002) Generating functions for computing the Myerson value. Ann Oper Res 109:143-158

Gallego I, Fernández JR, Jiménez-Losada A, Ordóñez M (2014) A Banzhaf value for games with fuzzy communication structure: computing the power of the political groups in the European Parliament. Fuzzy Sets Syst 255:128-145 
Gilles RP, Owen G, van den Brink R (1992) Games with permission structures. Int J Game Theory 20:277_ 293

Gillies DB (1953) Some theorems on $n$-person games. Ph.D. Thesis, Princeton University Press, Princeton

Herings PJJ, van der Laan G, Talman AJJ (2008) The average tree solution for cycle free games. Games Econ Behav 62:77-92

Herings PJJ, van der Laan G, Talman AJJ, Tang Z (2010) The average tree solution for games with communication structure. Games Econ Behav 68(2):626-633

Hobbs BF (1992) Using game theory to analyze electric transmission pricing policies in the United States. Eur J Oper Res 56(2):154-171

Jiménez-Losada A, Fernández JR, Ordó ñez M, Grabisch M (2010) Games on fuzzy communication structures with Choquet players. Eur J Oper Res 207:836-847

Jiménez-Losada A, Fernández JR, Ordó ñez M (2013) Myerson values for games with fuzzy communication structure. Fuzzy Sets Syst 213:74-90

Knuth DE (1976) Big omicron and big omega and big theta. ACM SIGACT News 8:18-24

Myerson R (1977) Graphs and cooperation in games. Math Oper Res 2:225-229

Shapley LS (1988) A value an $n$-person games. In: Kuhn HW, Tucker AW (eds) Contributions to the theory of games II. Princeton University Press, Princeton, pp 307-317

Tsurumi M, Tanino T, Inuiguchi M (2001) A Shapley function on a class of cooperative fuzzy games. Eur J Oper Res 129:596-618

Publisher's Note Springer Nature remains neutral with regard to jurisdictional claims in published maps and institutional affiliations. 